\title{
Solid State Backscattered Electron Detector Optimized for Minimum Detectable Energy and Maximum Scan Speed
}

\author{
A.Liebel*, H.Soltau*, R.Eckhardt*, O.Jaritschin**, A.Niculae**, F.Schopper*** \\ *PNSensor GmbH, Römerstr. 28, D-80803 München, Germany \\ **PNDetector GmbH, Emil-Nolde-Str.10, D-81735 München, Germany \\ ***MPI Halbleiterlabor, Otto-Hahn-Ring 6, D-81739 München, Germany
}

The measurement of Backscattered Electrons (BSE) in a Scanning Electron Microscope (SEM) is an important tool for compositional analysis. Latest techniques like Low Voltage Scanning Electron Microscopy (LVSEM) for the observation of insulating materials or biological samples demand for highly sensitive and fast detectors with low noise characteristics. Our aim is to fulfill these requirements by means of advanced Solid State Backscattered Electron Detectors, based on ultrapure silicon technology.

The sensitivity of electron detectors is defined by the dead layer of the detector entrance window. Recent measurements of the detector collection efficiency as a function of electron energy with our standard electron detectors and some test structures with optimized entrance window already showed large improvements in efficiency at electron energies below $10 \mathrm{keV}$ (see Fig. 1). We now implemented this technology into the production of our newest Backscattered Electron Detectors. Detailed results of the detector collection efficiency of these new detector chips with minimized dead layer will be presented and the gain in signal to noise ratio due to this advance will be shown.

The maximum attainable scan speed with solid state detectors is often limited due to bandwidth issues. However, detection speed can be increased by lowering the capacitance of the detector and it's connections to the electronics. We have introduced a concept with the preamplifier electronics integrated into the SEM chamber to minimize parasitic capacitances of the connection cables (see Fig. 2.a). The detector chips can be fully depleted which minimizes the signal capacitance to a level where the maximum scan speed is only determined by the bandwidth of the readout electronics. The dark current of the ultrapure silicon chips under these conditions is still lower than $100 \mathrm{pA}$ at room temperature, which is far less the measured signal noise currents of the detector, even at working conditions with very low beam voltages and currents. Images have been taken at pixel dwell times down to $100 \mathrm{~ns}$ without any smearing effects and with very low noise levels. Measurement results with further improvements of the signal capacitance will be presented.

Figure 2.b shows a mounted 4-quadrant annular detector with a total active area of $50 \mathrm{~mm}^{2}$ on a flat assembly which allows very small working distances down to $3 \mathrm{~mm}$ and hence, enables high resolution BSE images. The flexible flat cable and micro pitch connector support the feasibility of different custom assembly solutions like the direct integration into the pole piece of the microscope. The active area of our newest detectors has been increased while keeping the outer dimensions the same. This results in larger solid angles and therefore better signal to noise ratios. 


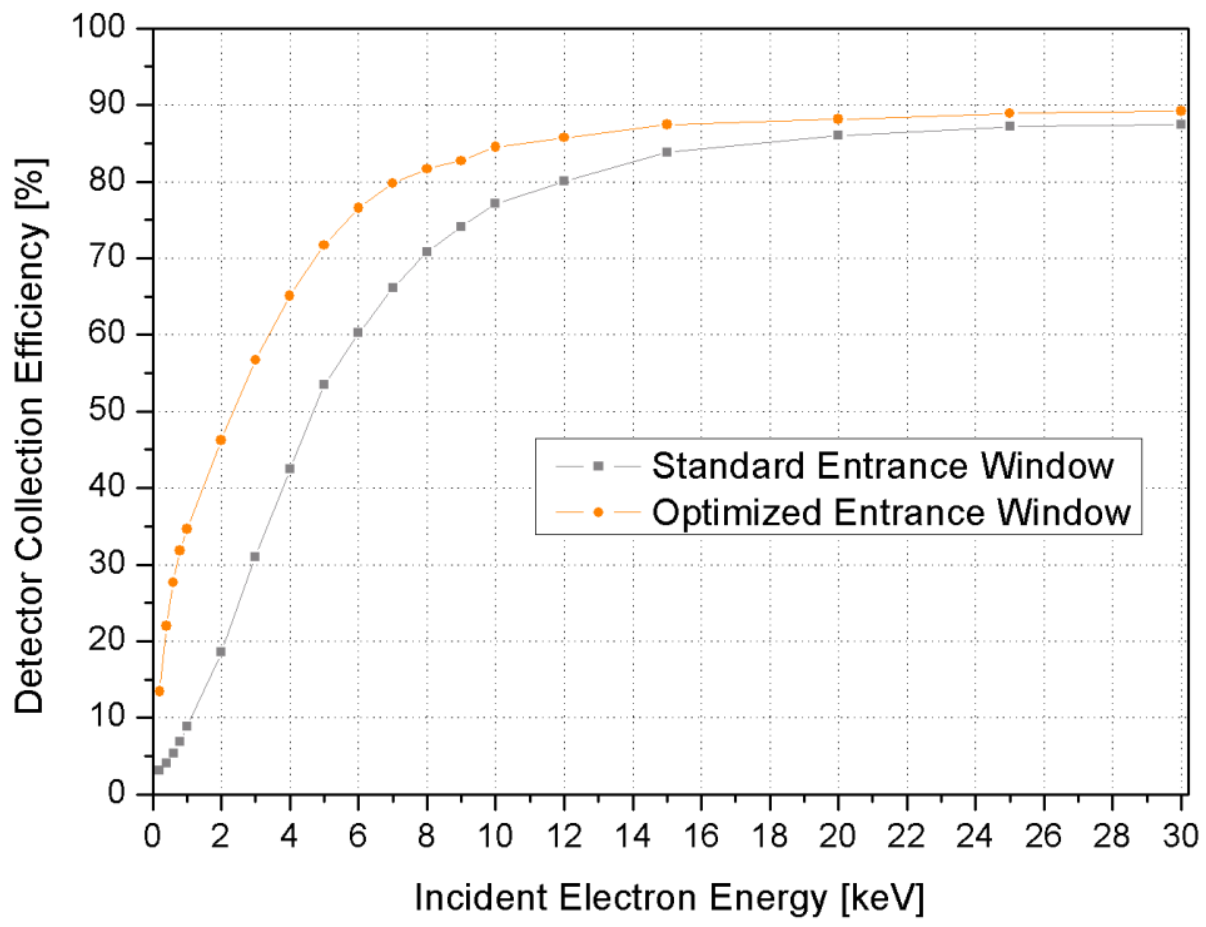

Fig. 1. Measured Detector Collection Efficiency as a function of energy. First optimizations of the detector entrance window already led to large improvements with an increase of more than $100 \%$ for energies below $3 \mathrm{keV}$.

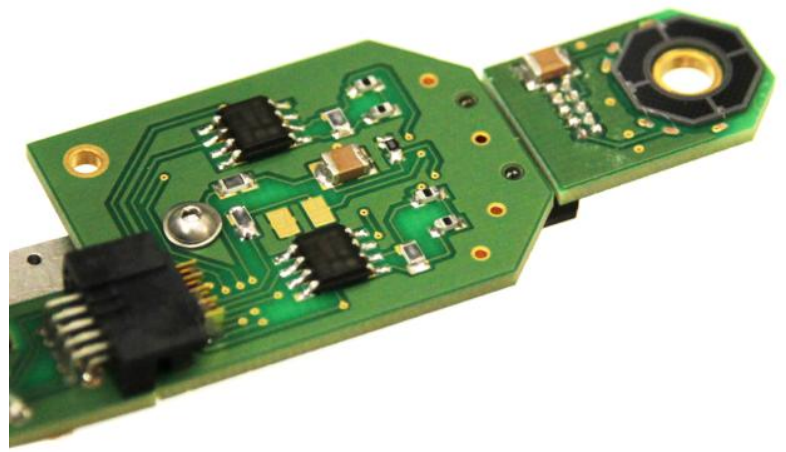

a)

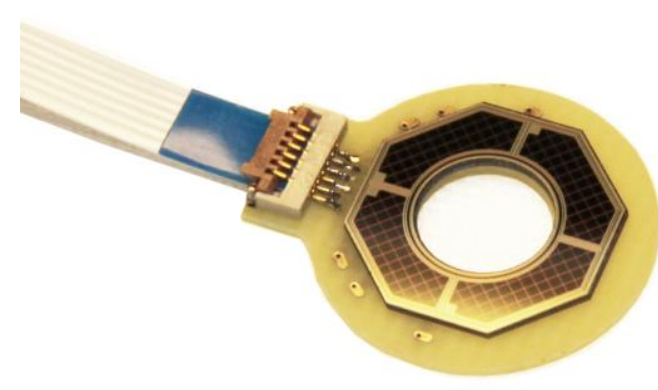

b)

Fig. 2. Mounted 4-Quadrant Backscattered Electron Detector Chip, (a) directly connected to the preamplifier electronics inside the SEM chamber to minimize signal capacitance and noise for the operation at high scan speeds and (b) a flat version on a flexible cable enabling minimum working distances. 